particles were similar in size, structure and composition. Nature Nanotech. http://doi.org/ rpg (2014)

\section{Stubborn microbe} finds hiding spots

Salmonella bacteria can escape antibiotics and immunesystem attack by hiding inside a host's immune cells.

Roland Regoes and Wolf-Dietrich Hardt of the Swiss Federal Institute of Technology in Zurich and their colleagues infected mice with Salmonella enterica and then treated the animals with the antibiotic ciprofloxacin. The team found that, after most of the infection had cleared from organs, about $10 \%$ of the Salmonella bacteria inside lymph nodes that drain the intestines were still viable and growing. These bacteria re-established infection after antibiotic treatment.

In a separate study, Sophie Helaine, David Holden, and their colleagues at Imperial College London found that Salmonella cells can also persist inside macrophages that ingest them. Molecules that stimulate certain types of immune cells could, in combination with antibiotics, improve treatment.

PLoS Biol. 12, e1001793 (2014);

Science 343, 204-208 (2014)

\section{ANIMAL BEHAVIOUR}

\section{Worms bond to} reach new heights

To reach passing beetles, parasitic worms congregate into towers up to 30 times taller than an individual.

Hans-Joachim Knölker at the Dresden University of Technology in Germany, Teymuras Kurzchalia at the Max Planck Institute of Molecular Cell Biology and Genetics in Dresden, and their team found that juvenile Pristionchus pacificus secrete a waxy substance, dubbed nematoil by the authors, that functions as an adhesive between animals. This allows them to form towers with up to 1,000 individuals, reaching as high as 1 centimetre, which can attach to a host.

Nature Chem. Bio. http://doi.org/ rqt (2014) \section{MATERIALS \\ Waste glass finds diffuse use}

A by-product of antiquated glass-making processes could find a new lease of life as an optical diffuser.

Manufacturers now avoid producing crystals, called devitrite, in their soda-limesilica glass because they degrade the material's optical qualities. But Haider Butt at the University of Birmingham, $\mathrm{UK}$, and his team show that this currently undesirable form of glass can act as a diffuser, owing to the fact that needlelike crystals of devitrite scatter light to wide angles of up to $120^{\circ}$. Because devitrite is both a highly efficient diffuser and cheap to produce, the authors say it could be used in applications such as medical lasers.

ACS Nano. http://doi.org/rpk (2014)

\section{PALAEONTOLOGY}

\section{Algae dealt blow to ancient whales}

The unearthing of more than 40 marine mammal fossils (pictured) at a site in Atacama, Chile, has revealed that they probably died en masse in four events due to toxic algae.

Nicholas Pyenson of the Smithsonian Institution in

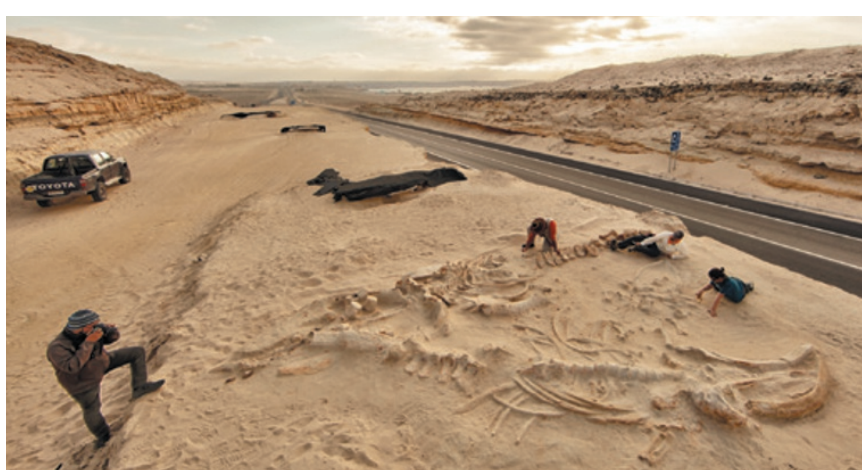

The most viewed papers in science

\section{Hopping DNA linked to schizophrenia}

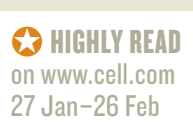

Mobile DNA elements activated by environmental or genetic triggers could boost susceptibility to schizophrenia.

Tadafumi Kato at the RIKEN Brain

Science Institute in Saitama, Japan, Kazuya Iwamoto at the University of Tokyo and their colleagues found higher copy numbers of a genetic element, called the $\mathrm{L} 1$ retrotransposon, in the DNA extracted from the post-mortem brains of patients with schizophrenia compared with that from the brains of healthy individuals. The authors also showed that the L1 elements tended to localize to genes linked to schizophrenia and neuronal synapses.

The same phenomenon was observed in mice and monkeys exposed perinatally to chemical stressors that are known to promote schizophrenia-like behaviours, and in cultured neurons derived from stem cells carrying a schizophreniarelated chromosomal deletion.

Neuron 81, 306-313 (2014)
Washington DC and his colleagues found that the fossils, including baleen and other whales, seals and an aquatic sloth, were arranged in four distinct layers, which are between 9 million and 6.5 million years old. The only modern event known to trigger such recurring and rapid die-offs of multiple species is extreme growth of toxic algae. The animals could have died after eating contaminated prey or have been directly affected by the algae's toxin. Other ocean areas that once created such blooms could harbour substantial fossil vertebrate remains, the authors say.

Proc. R. Soc. B 281, 20133316 (2014)

\section{Pheromone turns on goat brains}

Researchers have pinpointed a molecule produced by male goats that activates reproduction in females outside of their normal breeding season.

Yukari Takeuchi at the University of Tokyo and her colleagues used a special cap to capture 18 different pheromone molecules emitted from the heads of male goats. They then exposed females to the molecules, and used implanted electrodes to monitor activity in a brain region that regulates reproduction. One molecule, 4-ethyloctanal, triggered the biggest response of all the compounds tested.

This is the first molecule shown to stimulate a key regulator of reproduction in mammals called the gonadotropin releasing hormone pulse generator. Curr. Biol. http://doi.org/rqv (2014)

\section{DNATURE.COM}

For the latest research published by Naturevisit:

www.nature.com/latestresearch 